\title{
Investigation of the clinical features and therapeutic methods for the management of inflammatory lacrimal punctum diseases
}

\author{
Lin Ye ${ }^{1 \star}$, Bingzhi Yang ${ }^{2}$, Yuan Wang ${ }^{1}$, Hongbo Cheng ${ }^{1}$, Yun Peng ${ }^{1}$ and Jingxian \\ Zhang ${ }^{1}$ \\ ${ }^{1}$ Department of Lacrimal Apparatus Disease, ${ }^{2}$ Out-Patient Department, Shenzhen Eye Hospital, Shenzhen Key Laboratory of \\ Ophthalmology, Affiliated Shenzhen Eye Hospital of Jinan University, Shenzhen, China \\ *For correspondence: Email: liyeeesz@sina.com; zhangjxianer@sina.com
}

\begin{abstract}
Purpose: To establish if there are different classes of inflammatory lacrimal punctum diseases (ILPDs) and to examine the various strategies by which they can be managed therapeutically.

Methods: Two hundred and fifty nine (259) patients with inflammatory punctum lacrimal disease were identified and used as subjects for this study. Each patient was carefully examined for evidence of morphology of lacrimal punctum which was confirmed mainly by lacrimal duct flushing and probing. Appropriate therapeutic managements were adopted for patients with other inflammatory conditions besides ILPD. The clinical effects of the various therapeutic strategies were documented.

Results: Eighty-seven (87) patients out of the 259 (32.53\%) suffered from acute or chronic conjunctivitis while 66 patients $(5.61 \%)$ suffered from inflammatory lacrimal passage diseases. Patients with both conjunctivitis and lacrimal passage inflammation, patients with dry-eye symptoms, patients with just one of the conditions, and patients with mere evidence of superior punctalacrimalis represented 13.15, 14.19, 14.53, and $33.91 \%$, respectively. Mere evidence of inferior punctalacrimalis, and presence of acute inflammation were seen in 48.76 and $13.49 \%$ of the 259 patients, respectively, while those with chronic inflammation lasting for $2.97 \pm 0.13$ years, comprised $86.51 \%$. Antibiotic eye drops were used for acute inflammation, while chronic inflammation was treated with antibiotic eye drops, lacrimal punctum expansion, pus elimination, and punctum-sparing canaliculotomy. Both therapeutic methods produced satisfactory curative effects.

Conclusion: The results show that satisfactory therapy of lacrimal punctum inflammation can be achieved if the right therapeutic agents and procedures are adopted based on clinical characteristics of the ILPD manifesting in the patient.
\end{abstract}

Keywords: Lacrimal punctum, Inflammatory disease, Conjunctivitis, Dry-eye symptoms

Tropical Journal of Pharmaceutical Research is indexed by Science Citation Index (SciSearch), Scopus, International Pharmaceutical Abstract, Chemical Abstracts, Embase, Index Copernicus, EBSCO, African Index Medicus, JournalSeek, Journal Citation Reports/Science Edition, Directory of Open Access Journals (DOAJ), African Journal Online, Bioline International, Open-J-Gate and Pharmacy Abstracts

\section{INTRODUCTION}

The lacrimal punctum is located at the bitemporal of the inner canthus, at the junction of the ciliary and lacrimal parts of the superior and inferior palpebral margin, which is the initiating terminal of the lacrimal passage. Inflammatory disease obstructs the flow of tears into the lacrimal passage resulting in epiphora [1-4]. Presently, there is paucity of information in the literature on the clinical characteristics and pathological course of inflammatory lacrimal punctum disease. Previously, it was believed that inflammatory lacrimal punctum disease occurred 
along with conjunctivitis and canaliculitis, and that the severity is predicated on concomitant development of conjunctivitis and canaliculitis.

However, no specific study on lacrimal punctum appears to have been carried out with published results. Based on clinical observation, it is possible to know that inflammatory lacrimal punctum disease has specific features, and that in the course of the disease, it may or may not be associated with conjunctivitis or inflammation of other lacrimal passages. It is anticipated that the results obtained from the clinical observations in this study will form the basis for providing a general description of its occurrence, development, and treatment outcomes, thereby adding new information to our current knowledge of this disease. Furthermore the findings may provide information on therapeutic methods that may be explored in the management of different stages of puncta lacrimalis.

\section{METHODS}

Data from 259 patients with puncta lacrimalis (289 eyes) treated in the Department of Lacrimal Apparatus Disease of Shenzhen Eye Hospital from January 2008 to December 2013 were analyzed retrospectively. The 259 patients consisted of 131 males (with 165 infected eyes) and 128 females (with 142 infected eyes), with ages ranging from 13 to 82 (mean age $=51.8 \pm$ 5.31 years). In some patients, symptoms of the disease were noticed 2 weeks before reporting at the hospital whereas in others the time interval between the appearance of symptoms and hospital visitation ranged between three weeks to 20 years. The average time lapse from onset of symptoms to hospital visitation was $2.8 \pm 1.22$ years. Patients with congenital lacrimal passage abnormalities and patients who suffered from abnormal lacrimal punctum structures and functions due to certain trauma were excluded from this study. This study was approved by the ethics committee of the Affiliated Shenzhen Eye Hospital of Jinan University, Shenzhen, China (approval no. [2014]-JNU-005) and was carried out in accordance with the Guidelines of the World Medical Association Declaration of Helsinki [5].

\section{Study design}

Each patient's medical history was carefully documented. With the aid of slit lamp, the shape of the lacrimal punctum, position, and condition of the lacrimal caruncle, halfmoon wrinkle wall, palpebral conjunctiva, and bulbar conjunctiva were examined. Lacrimal duct flushing and probing, and other examinations were performed.
Pictures were taken with the slit-lamp microscope, and the characteristic changes observed during the course of the disease were recorded. Furthermore, appropriate therapeutic modalities were adopted for the management of specific case(s) and the observed outcomes documented.

\section{Statistical analysis}

Data analyses were performed using the SPSS 20.0 statistical software package. The data are presented as mean \pm standard deviation (SD). Student's $t$ test was used to evaluate significant differences between groups. $P<0.05$ was considered statistically significant.

\section{RESULTS}

Among the 259 patients (289 infected eyes) who suffered from inflammatory lacrimal punctum disease were 87 patients (94 infected eyes) comprising $32.53 \%$ of the infected eyes, with acute or chronic conjunctivitis. Sixty-nine patients (with 74 infected eyes) had other diseases of the lacrimal passage, such as canaliculitis and dacryocystitis. They constituted $25.61 \%$ of the total number of infected eyes. Thirty-four patients (38 infected eyes) suffered from both conjunctivitis and lacrimal passage inflammation, and constituted $13.15 \%$ of the total number of the infected eyes of patients included in the study. Thirty-two patients (with 41 infected eyes) suffered from dry-eyes and they formed $14.19 \%$ of the total number of infected eyes, while 37 patients (42 infected eyes) suffered from lacrimal punctum inflammation and constituted $14.53 \%$ of the total number of infected eyes.

Among all the patients who suffered from inflammatory lacrimal punctum disease, 93 (having 98 infected eyes) had superior puncta lacrimalis, which was $33.91 \%$ of the infected eyes, while 129 patients (141 infected eyes) had inferior puncta lacrimalis, and constituted 48.79 $\%$ of the infected eyes. Thirty-seven patients (50 infected eyes) suffered from both inferior and superior puncta lacrimalis, which translated to $17.30 \%$ of the infected eyes.

The patients who suffered from inflammatory lacrimal punctum disease were divided into acute inflammation and chronic inflammation groups according to the duration of the disease. There were 37 patients (with 39 infected eyes), and $13.49 \%$ of the total number of infected eyes had acute inflammation. The clinical manifestations included sudden and acute attacks, and the course of the disease was generally 4 weeks. All the patients also had redness of the eye, swelling 
and pain due to the diseased palpebral bulbar conjunctiva of the inner canthus, which in some cases involved the eyelid and skin of the inner canthus. The patients often experienced itching and flow of tears, and other symptoms in the inner canthus.

Physical signs of the disease were observed at the center of the lacrimal punctum aperture, the lacrimal punctum wall, and the surrounding palpebral conjunctiva centripetally, resulting in hyperemia and edema. In the marginal zones of the redness and swelling, the palpebral vasculature of the conjunctiva presented with plentiful and circuitous angiectasis (Figure 1A). Due to redness and swelling, the lacrimal punctum aperture showed a closed morphology, Generally, the surfaces of the affected eyes were coated with inflammation exudates. Seven patients (with 7 infected eyes) had acute canaliculitis, which presented as typical emulsion drop (Figure 1B). For these patients, antibiotic eye drops and symptomatic treatment were used to manage the associated conjunctivitis, canaliculitis [6], dacryocystitis, and dry-eye symptoms. After the redness and swelling gradually vanished, the lacrimal passage was flushed, and the inflammation eventually resolved completely.

There were 222 patients (250 infected eyes) with chronic inflammation that covered $86.51 \%$ of the eyes. For these patients, the onset of the disease was slow. The shortest course of the disease was 4 weeks, and the longest was 20 years, with an average of $2.97 \pm 0.13$ years. They also suffered from chronic conjunctivitis, which at times developed from acute inflammation.

The major symptoms were lacrimation and itching that was sensed in the inner canthus. Some of the patients presented with lacrimation when exposed to wind. With exacerbation of inflammation, the tears were accompanied by mucous secretion. During the initial period of inflammation, it presented as an acute attack, but if not treated, the illness gradually worsened. Type I puncta lacrimalis (lacrimal punctum centrality shrink type) was identified in 105 patients (120 infected eyes), comprising $48 \%$ of cases of chronic inflammation. In these patients, the palpebral conjunctiva was found to have developed from the four sides of the lacrimal punctum to the lacrimal punctum as the central portion of the lacrimal punctum shrank (Figure 2A).

Type II (vitreoretinopathy coverage type) was identified in 42 cases (47 infected eyes), which constituted $18.8 \%$ of those in the chronic inflammation group. Type II was evidenced by reduction and flatness of some parts of the papilla cyclic annular dam apophysis, ligule shape vitreoretinopathy of the palpebral conjunctiva access and covering of lacrimal punctum aperture from the flat position (Figure 2B). Type III was also identified (lacrimal punctum aperture close type) in 39 cases (44 infected eyes), which translated to $17.60 \%$ of all chronic inflammation cases. It presented as lack of hyperemia and edema, but the palpebral conjunctiva around the lacrimal punctum showed angiectasis along with neovascularization. The palpebral conjunctiva around the lacrimal punctum aperture presented with proliferation and hypertrophy, embedding the lacrimal punctum wall and obstructing the lacrimal punctum aperture.

However, when the fleshy and proliferative conjunctiva were removed, the lacrimal punctum aperture could be seen (Figure 2C). Type IV (lacrimal punctum pus embedding type) was
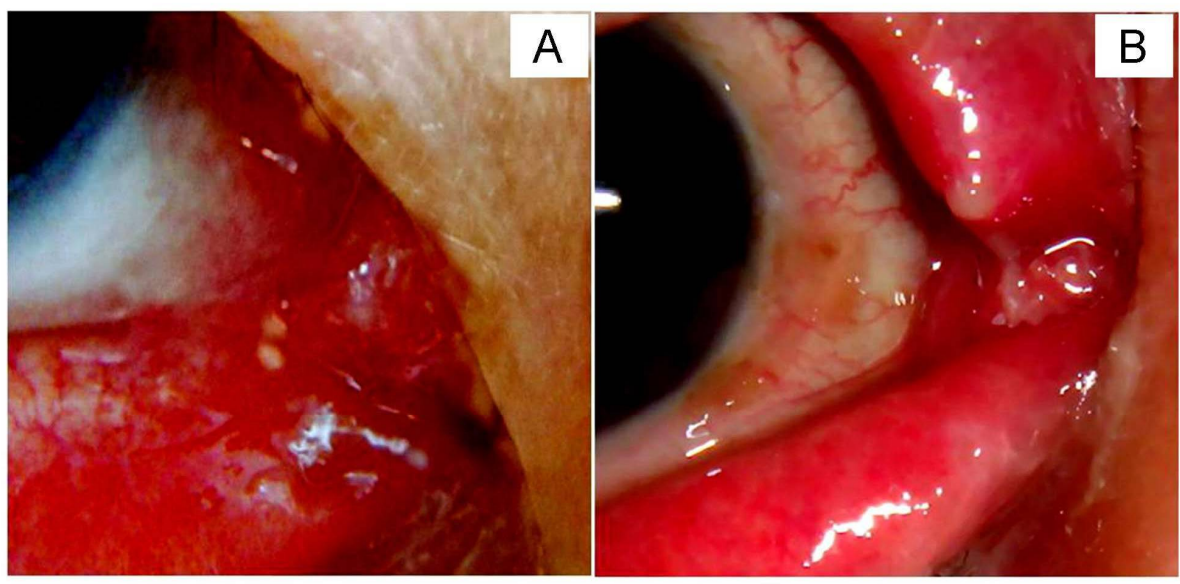

Figure 1: Acute lacrimal punctum inflammation $(A=$ Redness and swelling due to the palpebral bulbar conjunctiva of inner canthus; $\mathrm{B}=$ Emulsion droplet shape) 
identified in 25 cases (27 infected eyes), which constituted $10.80 \%$ of chronic inflammation cases. It presented as proliferating palpebral conjunctiva wrapping around the thick pus and lacrimal punctum aperture, forming a white molehill-like protrusion on the lower eyelid (Figure 2D).Type $\mathrm{V}$ (lacrimal punctum vanished type) was also identified in 11 cases (12 infected eyes), comprising $4.8 \%$ of the chronic inflammation cases. It presented as atrophy of the lacrimal punctum wall and surrounding tissues. The cyclic annular dam tissue was flat, and the lacrimal punctum aperture could not be entirely seen (Figure 2E).

For each of the above 5 groups of patients, different methods of therapy were applied. In type I group of patients, antibiotic eye drops were used and lacrimal passage expansion was performed with lacrimal puncta expander. For the type II group, antibiotic eye drops were used, and after one week of usage shrinkage of parts of the patients' vitreoretinopathy occurred. In cases where vitreoretinopathy still covered the lacrimal punctum aperture, resection of the parts of the vitreoretinopathy that covered the lacrimal punctum was done, followed by "lacrimal punctum expansion". For the type III group, antibiotic eye drops were used first, and thereafter lacrimal punctum angioplasty was performed. For the type IV group, resection of the encapsulated mucosal tissue was done to release the tenuous fester, and this was followed by angioplasty of the lacrimal punctum. After the therapies, patients' puncta lacrimalis were no longer evident and the lacrimal punctum assumed the normal shape.

\section{DISCUSSION}

The lacrimal punctum is the entrance of the tear excretory system to the eye. Under normal circumstances, the superior lacrimal punctum is located $6.0 \mathrm{~mm}$ inferior and posterior from the bitemporal site of the inner canthus. The inferior lacrimal punctum is located $6.5 \mathrm{~mm}$ superior and posterior from the bi-temporal site of the inner canthus. The normal lacrimal punctum diameter is 0.2 to $0.3 \mathrm{~mm}$, but it expands slightly with age. The lacrimal punctum is the open-end of the lacrimal canaliculus similar to the mouth of a well, which is open and bare on the surface of palpebral conjunctiva. It consists of the lacrimal punctum mouth, the lacrimal punctum wall and the lacrimal punctumpapilla (Figure 3 ).

On the surface of the periphery of the lacrimal punctum wall, the palpebral conjunctiva is thick, presenting as a dam apophysis, which is called the lacrimal punctum papilla. Within its deep layer is the lacrimal canaliculus, which is surrounded by the lacrimal punctum sphincter.
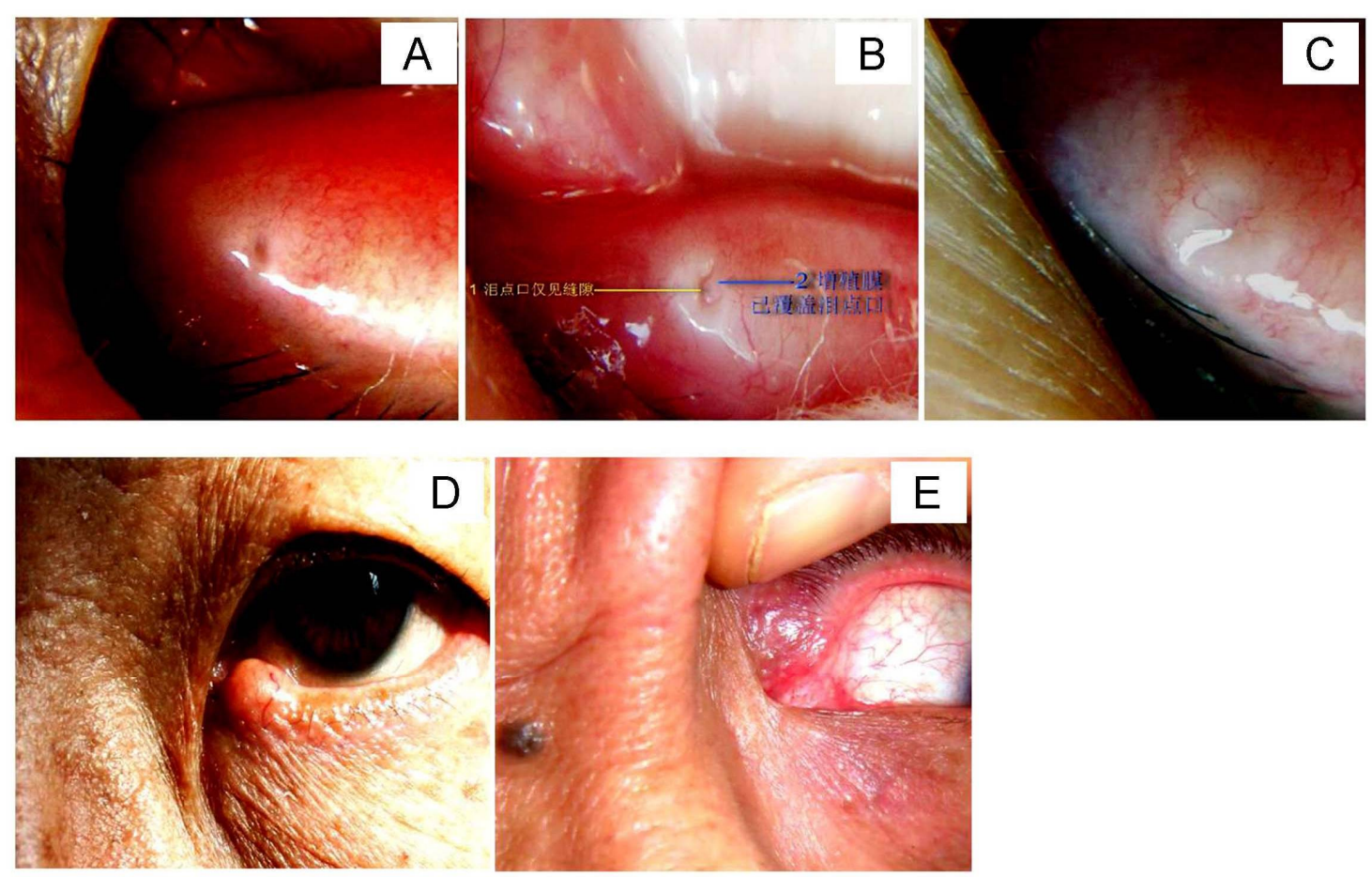

Figure 2: Chronic lacrimal punctum inflammation $(A=$ Type 1 Lacrimal punctum type with shrinkage of the central portion; $B=$ Type 2 Lacrimal punctum vitreoretinopathy coverage type; $C=$ Type 3 Lacrimal punctum mouth close type; $\mathrm{D}=$ Type 4 Lacrimal punctum pus embedding type $\mathrm{E}=$ Type 5 Lacrimal punctum vanishing type) 


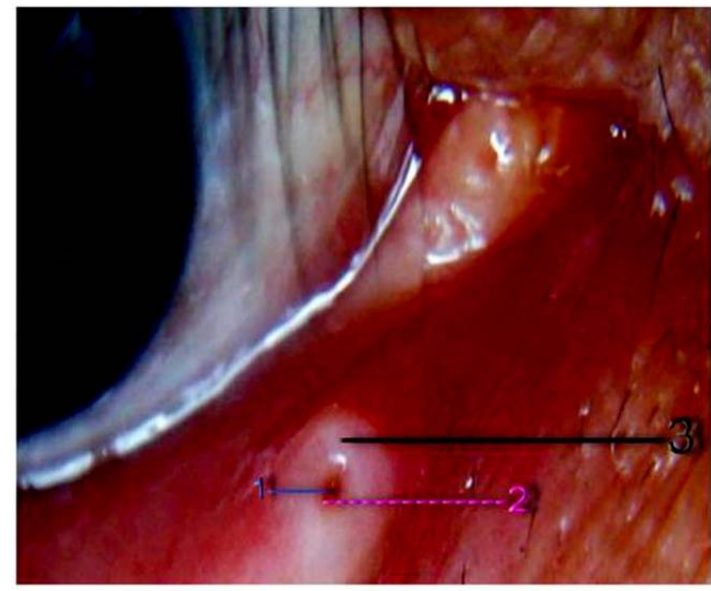

Figure 3: Lacrimal punctum (1 =. Lacrimal punctum mouth; 2 =. Lacrimal punctum wall; $3=$.Lacrimal punctum papilla)

Under normal circumstances, the lacrimal punctum aperture fits closely on the surface of the eyeball. It is located at the bottom of the tear lake. When the eyelid closes and opens, the superior lacrimal punctum slides into the shallow trench between the conjunctiva half-moon wrinkle wall and lacrimal caruncle, and the inferior lacrimal punctum slides into the shallow trench between the half-moon wrinkle wall and the eyeball.

The physiological functions of the lacrimal punctum includes absorbing tears into the lacrimal canaliculus and maintaining and stabilizing the normal cycle of tears on the surface of the eyeball through the movement of the lacrimal punctum sphincter. Due to the anatomical position of the lacrimal punctum, it is easily infected. Thus conjunctivitis and canaliculitis may spread to the lacrimal punctum [1]. Inflammatory lacrimal punctum disease and its clinical characteristics and disease the right therapeutic methods were examined in this study. In 259 cases (289 eyes) of lacrimal punctum inflammatory disease, patients who suffered from acute or chronic conjunctivitis comprised $32.53 \%$ of the cases, those who suffered from other inflammatory processes of the lacrimal passage comprised $25.61 \%$, and those with both conjunctivitis and lacrimal passage inflammation comprised $13.15 \%$. The data in our study is consistent with the previous published study [8]. The reason for this is that the conjunctiva, lacrimal punctum, lacrimal canaliculus, and lacrimal sac are connected to each other.

The inflammation of the conjunctiva, lacrimal canaliculus, and lacrimal sac may affect the lacrimal punctum, and their disease courses are more or less the same. This is probably why inflammatory lacrimal punctum disease has not received enough attention in clinical practice. Some patients with dry eyes were identified in this study. These patients generally will have recurrence within 1 - 2 years of the first dry-eye symptoms [9-11]. During the initial stages, their symptoms were off and on but when the course of the disease become protracted, the lacrimal punctum may be blocked. These patients generally need to be treated with lacrimal implants, which undoubtedly will enhance lacrimal punctum function.

However lacrimal punctum implants that should be used are the ones that will not irritate the lacrimal punctum when treating dry-eyes. In addition, during therapy efforts should be made to ascertain whether a patient is wearing an implant or not. Patients suffering from inflammatory lacrimal punctum disease, but who had puncta lacrimalis alone were among the patients included in this study. In these patients it is likely that the conjunctiva and lacrimal canaliculus are affected. However, clinicians usually neglect the diagnosis and therapy of idiopathic puncta lacrimal inflammation and focus on the treatment for conjunctivitis and canaliculitis only.

Of all the patients suffering from inflammatory lacrimal punctum disease, patients who merely presented with superior puncta lacrimalis were $33.91 \%$, and those who presented with inferior lacrimal punctum were $48.79 \%$. The present study provided the similar findings compared to the previous published studies [12,13]. The high percentage of the latter may be due to the low position of inferior lacrimal punctum, where tears and other pathogenic factors collecting.

It is likely that the disease worsens from Type I to Type V. At the onset of the disease, the mouth of the lacrimal punctum presents with central shrinkage due to the swelling. If untreated, the palpebral conjunctiva swelling will aggravate and gradually cover the mouth of the lacrimal punctum, which presents as vitreoretinopathy coverage type. The vitreoretinopathy will eventually cover the lacrimal punctum entirely, but after treatment, the lacrimal punctum mouth can be seen. Then, with worsening of the disease, pus is wrapped in the lacrimal punctum mouth, resulting in the pus embedding type. Finally, complete resolution of the lacrimal punctum represents the lacrimal punctum vanished type. 
Based on the different disease courses, selected antibiotic eye drops, lacrimal punctum expansion, clearing of pus, lacrimal punctum shaping, and other treatments that were adopted produced satisfactory cures.

\section{Limitations of the study}

Although this study revealed some interesting results, there are also a few limitations. First, although 259 patients with inflammatory puncta lacrimalis disease were involved in this study, the sample size is considered small. Second, the outcomes could not be fully analyzed. Third, it was difficult to access the right drugs normally used for the therapeutic management of inflammatory puncta lacrimalis disease.

\section{CONCLUSION}

Lacrimal punctum inflammation is a disease of distinct clinical manifestations with clearly defined course of development. The findings of the study indicate that satisfactory therapy of the inflammation can be achieved if the right therapeutic agents and procedures are adopted based on the clinical characteristics of the ILPD manifesting in the patient.

\section{DECLARATIONS}

\section{Acknowledgement}

This work was supported by grants from Shenzhen Science and Technology Innovation Committee (Grant no. JCYJ20140415174819504) to LY Shenzhen "Sanming" Project, International Cooperation Project of Shenzhen Science and Technology Innovation Committee (Grant no. GJHZ20150316150106271) and Scientific Research of Guangdong Provincial Bureau of traditional Chinese Medicine (Grant no. 20171239).

\section{Conflict of Interest}

No conflict of interest associated with this work.

\section{Contribution of Authors}

The authors declare that this work was done by the authors named in this article and all liabilities pertaining to claims relating to the content of this article will be borne by them.

\section{Open Access}

This is an Open Access article that uses a funding model which does not charge readers or their institutions for access and distributed under the terms of the Creative Commons Attribution License (http://creativecommons.org/ licenses/by/ 4.0) and the Budapest Open Access Initiative (http://www.budapestopena ccessinitiative.org/read), which permit unrestricted use, distribution, and reproduction in any medium, provided the original work is properly credited.

\section{REFERENCES}

1. Bukhari A. Etiology of tearing in patients seen in an oculoplastic clinic in Saudi Arabia. Middle East Afr $J$ Ophthalmol 2013; 20(3): 198-200.

2. Carter KD, Nelson CC, Martonyi CL. Size variation of the lacrimal punctum in adults. Ophthal Plast Reconstr Surg 1988; 4(4): 231-233.

3. Freedman JR, Markert MS, Cohen AJ. Primary and secondary lacrimal canaliculitis: a review of literature. Surv Ophthalmol, 2011; 56(4): 336-347.

4. Takahashi Y, Kakizaki H, Nakano T, Asamoto K, Ichinose A, Iwaki M. Anatomy of the vertical lacrimal canaliculus and lacrimal punctum: a macroscopic study. Ophthal Plast Reconstr Surg 2011; 27(5): 384-386.

5. World Medical Association. World Medical Association Declaration of Helsinki: ethical principles for medical research involving human subjects. JAMA 2013; 310(20): 2191-2194.

6. Khu J, Mancini R. Punctum-sparing canaliculotomy for the treatment of canaliculitis. Ophthal Plast Reconstr Surg 2012; 28(1): 63-65.

7. Chen D, Li N, Wan $P$, Xiao J, Liiu Y, Wang $X$, Wang $Z$. Novel procedure for treating canalicular obstruction by re-canaliculisation and bicanalicular intubation. $\mathrm{Br} J$ Ophthalmol, 2012; 96(3): 366-369.

8. Batth SS, Sreeraman R, Dienes E, Beckett LA, Daly ME, Cui J, Mathai M, Purdy JA, Chen AM. Clinical-dosimetric relationship between lacrimal gland dose and ocular toxicity after intensity-modulated rediotherapy for sinonasal tumors. $\mathrm{Br} J$ Radiol 2013; 86(1032): 20130459.

9. Chen F, Wang J, Chen W, Shen M, Xu S, Lu F. Upper punctal occlusion versus lower punctal occlusion in dry eye. Invest Ophthalmol Vis Sci2010; 51(11): 5571-5577.

10. Holzchuh R, Villa Albers MB, Osaki TH, Igami TZ, Santo RM, Kara-Jose N, Holzchuh N, Hida RY. Two-year outcome of partial lacrimal punctal occlusion in the management of dry eye related to Sjogren syndrome. Curr Eye Res, 2011; 36(6): 507-512.

11. Kakizaki H, Takahashi Y, Iwaki M, Nakano T, Asamoto K, Ikeda H, Goto E, Selva D, Leibovitch I. Punctal and canalicular anatomy: implications for canalicular occlusion in severe dry eye. Am J Ophthalmol, 2012; 153(2): 229-237 e221. 
12. Huang $D$, Luo $Q$, Yang $H$, Mao $Y$. Changes of lacrimal gland and tear inflammatory cytokines in thyroidassociated ophthalmopathy. Invest Ophthalmol Vis Sci 2014; 55(8): 4935-4943.
13. Mito $H$, Takahashi $Y$, Nakano $T$, Asamoto $K$, Ikeda $H$, Kakizaki H. Consecutive microscopic anatomical characteristics of the lacrimal asc and nasolacrimal duct: cases with or without inflammation. Invest Ophthalmol Vis Sci 2014; 55(8): 5233-5237. 